\title{
CONVOLUTION SEMIGROUPS OF STATES
}

\author{
J. MARTIN LINDSAY AND ADAM G. SKALSKI
}

\begin{abstract}
Convolution semigroups of states on a quantum group form the natural noncommutative analogue of convolution semigroups of probability measures on a locally compact group. Here we initiate a theory of weakly continuous convolution semigroups of functionals on a $C^{*}$-bialgebra, the noncommutative counterpart of a locally compact semigroup. On locally compact quantum groups we obtain a bijective correspondence between such convolution semigroups and a class of $C_{0}$-semigroups of maps which we characterise. On $C^{*}$-bialgebras of discrete type we show that all weakly continuous convolution semigroups of states are automatically norm-continuous. As an application we deduce a known characterisation of continuous conditionally positive-definite Hermitian functions on a compact group.
\end{abstract}

\section{INTRODUCTION}

Convolution semigroups of probability measures on locally compact groups, and semigroups, play an important role in both probability theory and functional analysis. They underpin all approaches to stochastic processes with independent identically distributed increments, that is Lévy processes ([Ber]). The analysis of convolution semigroups is also an independent area of research, well documented in the monograph [Hey]. Given the recent development of a satisfactory theory of locally compact quantum groups $([\mathrm{KuV}])$ it is natural to investigate convolution semigroups of states on such objects - states being the noncommutative counterpart of probability measures.

We recently introduced the notion of quantum Lévy process on a locally compact quantum semigroup, in other words a $C^{*}$-bialgebra $\left(\left[\mathrm{LS}_{2}\right]\right)$. As in the classical theory of Lévy processes, and the earlier noncommutative theory of Lévy processes on purely algebraic quantum semigroups ([Sch]), the concept of a convolution semigroup of states plays a fundamental role here too. In our papers the strongest results are obtained under the assumption that the Markov semigroup of the process is norm-continuous. By one form of analogy, the class of processes investigated there may thereby be viewed as a quantum analogue of the class of compound Poisson processes.

The present paper forms the first step in analysing general quantum Lévy processes in the analytic context. We initiate a theory of weakly continuous convolution semigroups of functionals on locally compact quantum semigroups. In particular we prove that, on $C^{*}$-bialgebras enjoying a 'residual vanishing at infinity' propertywhich is satisfied by all locally compact quantum groups, every weakly continuous convolution semigroup of functionals induces a $C_{0}$-semigroup on the algebra. From a probabilistic viewpoint this amounts to a Feller property ([Jac])). We give several characterisations of the resulting class of $C_{0}$-semigroups. We also prove that on $C^{*}$ bialgebras of discrete type weak continuity for a convolution semigroup of states 
implies norm-continuity. Here slice maps for non-normal functionals, in the sense of Tomiyama ([Tom]), play a crucial role. Our results show that all quantum Lévy processes on a discrete quantum semigroup are automatically of the type thoroughly studied in $\left[\mathrm{LS}_{2}\right]$. As an application we prove a result of Guichardet, characterising the continuous conditionally positive-definite Hermitian functions on a compact group ([Gui]).

\section{Preliminaries}

Given $C^{*}$-algebras $\mathrm{A}$ and $\mathrm{C}$, their spatial/minimal (respectively algebraic) tensor product will be denoted $A \otimes C$ (resp. $A \odot C$ ) and the multiplier algebra of $A$ will be denoted by $M(\mathrm{~A})$. The multiplier algebra is equipped with the strict topology, with respect to which the unit ball of $\mathrm{A}$ is dense in that of $M(\mathrm{~A})$. A linear map $T$ from A (or $M(\mathrm{~A})$ ) to $M(\mathrm{C})$ is strict if it is bounded and strictly continuous on bounded subsets of A (respectively $M(\mathrm{~A})$ ). Standard examples of strict maps include *homomorphisms which are nondegenerate (that is $T(\mathrm{~A}) \mathrm{C}$ is total in $\mathrm{C}$ ), continuous linear functionals on $\mathrm{A}$ and, more generally, slice maps of the type $\mu \otimes \mathrm{id}_{\mathrm{C}}: \mathrm{A} \otimes \mathrm{C} \rightarrow$ C $\subset M(\mathrm{C})$, where $\mu \in \mathrm{A}^{*}$. The collection of strict linear functionals on $M(\mathrm{~A})$ forms a closed subspace of $M(\mathrm{~A})^{*}$ which we denote by $M(\mathrm{~A})_{\beta}^{*}$. An important property of strict maps $T: \mathrm{A} \rightarrow M(\mathrm{C})$ is that they possess a unique strict extension $\widetilde{T}: M(\mathrm{~A}) \rightarrow M(\mathrm{C})$; the extension procedure does not change the norm (or cb-norm if $T$ is completely bounded) and preserves (complete) positivity. In particular, strict extension defines an isometric isomorphism $\mathrm{A}^{*} \rightarrow M(\mathrm{~A})_{\beta}^{*}$ mapping states to states. A strict map $T$ is called preunital if its strict extension is unital: $\widetilde{T}(1)=1$. Thus all states are preunital, as are nondegenerate ${ }^{*}$-homomorphisms. The existence and uniqueness of extensions allows composition of strict maps $T: \mathrm{A}_{1} \rightarrow M\left(\mathrm{~A}_{2}\right)$ and $S: \mathrm{A}_{2} \rightarrow M\left(\mathrm{~A}_{3}\right)$, with the resulting map $\mathrm{A}_{1} \rightarrow M\left(\mathrm{~A}_{3}\right)$ being strict. Finally, for strict completely positive maps $T_{i}: \mathrm{A}_{i} \rightarrow \mathrm{C}_{i}(i=1,2)$, their tensor product map $T_{1} \otimes T_{2}: \mathrm{A}_{1} \otimes \mathrm{A}_{2} \rightarrow M\left(\mathrm{C}_{1}\right) \otimes M\left(\mathrm{C}_{2}\right) \subset M\left(\mathrm{C}_{1} \otimes \mathrm{C}_{2}\right)$, is strict. For more information on multiplier algebras, strict maps, their tensor products and extensions, we refer to $[\mathrm{Lan}],\left[\mathrm{Ku}_{1}\right]$ and $\left[\mathrm{LS}_{2}\right]$.

By a semigroup on $\mathrm{A}$, or $M(\mathrm{~A})$, we mean a one-parameter family of bounded operators $\left(P_{t}\right)_{t>0}$ satisfying $P_{s+t}=P_{s} P_{t}$ and $P_{0}=\mathrm{id}_{\mathrm{A}}$ (no continuity in $t$ is assumed a priori). We call it a strict/completely bounded/completely positive semigroup if each map $P_{t}$ has that property. By elementary semigroup theory ([Dav], Proposition 1.23), $\left(P_{t}\right)_{t \geq 0}$ is a $C_{0}$-semigroup, i.e. it is strongly continuous in $t$, if it is weakly continuous, that is $t \mapsto \mu\left(P_{t}(a)\right)$ is continuous for each $a \in \mathrm{A}$ and $\mu \in \mathrm{A}^{*}$, at $t=0$.

We shall need two general results, in Sections 2 and 4 respectively. The first concerns the preservation of pointwise convergence under strict extension.

Proposition 1.1. Let $\left(\omega_{\lambda}\right)_{\lambda \in \Lambda}$ be a net of states on a $C^{*}$-algebra A converging pointwise to a state $\omega$ on $\mathrm{A}$. Then $\left(\widetilde{\omega}_{\lambda}\right)_{\lambda \in \Lambda}$ converges pointwise to $\widetilde{\omega}$.

Proof. Let $m \in M(\mathrm{~A})$ and let $\left(e_{i}\right)_{i \in \mathcal{I}}$ be an approximate unit for A. Then, for each $i \in \mathcal{I}$,

$$
\widetilde{\omega}_{\lambda}\left(\left(1-e_{i}\right)^{2}\right)=1+\omega_{\lambda}\left(e_{i}^{2}-2 e_{i}\right) \underset{2}{\stackrel{\lambda}{\longrightarrow}} 1+\omega\left(e_{i}^{2}-2 e_{i}\right)=\widetilde{\omega}\left(\left(1-e_{i}\right)^{2}\right)
$$


and so, by the Cauchy-Schwarz inequality for states,

$$
\begin{aligned}
\left|\left(\widetilde{\omega}_{\lambda}-\widetilde{\omega}\right)(m)\right| & \leq\left|\widetilde{\omega}_{\lambda}\left(m\left(1-e_{i}\right)\right)\right|+\left|\widetilde{\omega}\left(m\left(1-e_{i}\right)\right)\right|+\left|\left(\omega_{\lambda}-\omega\right)\left(m e_{i}\right)\right| \\
& \leq\|m\|\left(\widetilde{\omega}_{\lambda}\left(\left(1-e_{i}\right)^{2}\right)\right)^{\frac{1}{2}}+\|m\|\left(\widetilde{\omega}\left(\left(1-e_{i}\right)^{2}\right)\right)^{\frac{1}{2}}+\left|\left(\omega_{\lambda}-\omega\right)\left(m e_{i}\right)\right| \\
& \stackrel{\lambda}{\longrightarrow} 2\|m\| \widetilde{\omega}\left(\left(1-e_{i}\right)^{2}\right)^{1 / 2} .
\end{aligned}
$$

Since $\left(\left(1-e_{i}\right)^{2}\right)_{i \in \mathcal{I}}$ converges strictly to 0 , the result follows.

Remark. Using Kadison-Schwarz, instead of Cauchy-Schwarz, the above proof remains valid for strict completely positive preunital maps into the multiplier algebra of another $C^{*}$-algebra $\left(T_{\lambda}: \mathrm{A} \rightarrow M(\mathrm{C})\right)_{\lambda \in \Lambda}$ converging pointwise to another such $\operatorname{map} T$.

The following observation is due to Tomiyama ([Tom]); we include its elementary proof for the convenience of the reader. The notation $\bar{\otimes}$ is used for ultraweak tensor products of von Neumann algebras and ultraweakly continuous slice maps $([\mathrm{KaR}])$.

Proposition 1.2. Let $\mathrm{M}=\mathrm{M}_{1} \bar{\otimes} \mathrm{M}_{2}$, for von Neumann algebras $\mathrm{M}_{1}$ and $\mathrm{M}_{2}$, and let $\nu \in\left(\mathrm{M}_{1}\right)^{*}$. Then there is a unique map $\nu \otimes_{\mathrm{M}} \mathrm{id}_{2}: \mathrm{M} \rightarrow \mathrm{M}_{2}$ satisfying

$$
\varphi \circ\left(\nu \otimes_{\mathrm{M}} \operatorname{id}_{2}\right)=\nu \circ\left(\operatorname{id}_{1} \bar{\otimes} \varphi\right), \quad \varphi \in\left(\mathrm{M}_{2}\right)_{*},
$$

where $\mathrm{id}_{i}$ denotes the identity map on $\mathrm{M}_{i}(i=1,2)$. Moreover, $\nu \otimes_{\mathrm{M}} \mathrm{id}_{2}$ is a bounded operator of norm $\|\nu\|$.

Proof. Let $x \in \mathrm{M}$. Then, for $\varphi \in\left(\mathrm{M}_{2}\right)_{*}$,

$$
\left|\nu\left(\left(\operatorname{id}_{1} \bar{\otimes} \varphi\right)(x)\right)\right| \leq\|\nu\|\left\|\operatorname{id}_{1} \bar{\otimes} \varphi\right\|\|x\|=\|\nu\|\|\varphi\|\|x\|
$$

so the prescription $\varphi \mapsto \nu\left(\left(\operatorname{id}_{1} \bar{\otimes} \varphi\right)(x)\right)$ defines a bounded linear functional on $\left(\mathrm{M}_{2}\right)_{*}$ of norm at most $\|\nu\|\|x\|$. Therefore, invoking the canonical identification of $\left(\left(\mathrm{M}_{2}\right)_{*}\right)^{*}$ with $\mathrm{M}_{2}$, there is a bounded operator $\nu \otimes_{\mathrm{M}} \mathrm{id}_{2}: \mathrm{M} \rightarrow \mathrm{M}_{2}$, of norm at most $\|\nu\|$, satisfying (1.1). Since $\nu \otimes_{\mathrm{M}} \mathrm{id}_{2}$ clearly extends the map $\nu \otimes \mathrm{id}_{2}$ : $\mathrm{M}_{1} \otimes \mathrm{M}_{2} \rightarrow \mathrm{M}_{2}$, which has norm $\|\nu\|$, it follows that $\left\|\nu \otimes_{\mathrm{M}} \mathrm{id}_{2}\right\|=\|\nu\|$.

Remarks. By uniqueness, and the fact that $\left(\mathrm{M}_{2}\right)_{*}=\left\{\left.\phi\right|_{\mathrm{M}_{2}}: \phi \in B(\mathrm{~h})_{*}\right\}$ where $\mathrm{h}$ is the Hilbert space on which $\mathrm{M}_{2}$ acts, it follows that $\nu \otimes_{\mathrm{M}} \mathrm{id}_{2}=\left.\nu \otimes_{\mathrm{M}} \mathrm{id}_{B\left(\mathrm{~h}_{2}\right)}\right|_{\mathrm{M}}$. In particular, by Remark 1.4 of [LiW], $\nu \otimes_{\mathrm{M}} \mathrm{id}_{2}$ is completely bounded with cb-norm equal to $\|\nu\|_{\mathrm{cb}}=\|\nu\|$; moreover the map $\nu \otimes_{\mathrm{M}} \mathrm{id}_{2}$ is completely positive if the functional $\nu$ is positive. In fact, the above result still holds if $\nu$ is replaced by a completely bounded map into another von Neumann algebra $\mathrm{M}_{3}$, with (1.1) now reading $\left(\mathrm{id}_{3} \bar{\otimes} \varphi\right)\left(\nu \otimes_{\mathrm{M}} \mathrm{id}_{2}\right)=\nu \circ\left(\mathrm{id}_{1} \bar{\otimes} \varphi\right)([\mathrm{LiW}])$.

If $\nu$ is ultraweakly continuous then $\nu \otimes_{\mathrm{M}} \mathrm{id}_{2}$ equals $\nu \bar{\otimes} \mathrm{id}_{2}$. Of course there are also bounded operators $\operatorname{id}_{1} \mathrm{M} \otimes \mu: \mathrm{M} \rightarrow \mathrm{M}_{1}$, for each $\mu \in\left(\mathrm{M}_{2}\right)^{*}$, and the validity of the commutation relation

$$
\nu_{1} \circ\left(\mathrm{id}_{1} \mathrm{M} \otimes \nu_{2}\right)=\nu_{2} \circ\left(\nu_{1} \otimes_{\mathrm{M}} \mathrm{id}_{2}\right)
$$

for functionals $\nu_{i} \in\left(\mathrm{M}_{i}\right)^{*}, i=1,2$, naturally arises. Under the assumptions that $\mathrm{M}_{2}$ is infinite dimensional and the Hilbert space on which $\mathrm{M}_{1}$ acts is separable, Tomiyama showed that (1.2) holds for all $\nu_{2} \in\left(\mathrm{M}_{2}\right)^{*}$ if and only if $\nu_{1}$ is ultraweakly continuous, that is $\nu_{1} \in\left(\mathrm{M}_{1}\right)_{*}$ ([Tom], Theorem 5.1). There is a corresponding result for completely bounded maps ([Neu], Theorem 5.4). 


\section{Convolution semigroups on $C^{*}$-Bialgebras}

We first recall the definition.

Definition. A (counital) $C^{*}$-bialgebra is a $C^{*}$-algebra B equipped with a nondegenerate $*$-homomorphism $\Delta: \mathrm{B} \rightarrow M(\mathrm{~B} \otimes \mathrm{B})$, called the coproduct, and a character $\epsilon: \mathrm{B} \rightarrow \mathbb{C}$, called the counit, satisfying the coassociativity and counital properties:

$$
\left(\mathrm{id}_{\mathrm{B}} \otimes \Delta\right) \Delta=\left(\Delta \otimes \mathrm{id}_{\mathrm{B}}\right) \Delta \text { and }\left(\mathrm{id}_{\mathrm{B}} \otimes \epsilon\right) \Delta=\mathrm{id}_{\mathrm{B}}=\left(\epsilon \otimes \mathrm{id}_{\mathrm{B}}\right) \Delta .
$$

A useful neutral expression for the first two maps is $\Delta^{(2)}$. $C^{*}$-bialgebras are also called locally compact quantum semigroups; unital $C^{*}$-bialgebras are called compact quantum semigroups, or $C^{*}$-bialgebras of compact type.

Fix now, and for the rest of the paper, a $C^{*}$-bialgebra B. For $\lambda, \mu \in \mathrm{B}^{*}$, their convolution is defined as the following composition of strict maps:

$$
\lambda \star \mu=(\lambda \otimes \mu) \Delta .
$$

Convolution may also be viewed as a product on $M(\mathrm{~B})_{\beta}^{*}$.

Proposition 2.1. Both $\left(\mathrm{B}^{*}, \star\right)$ and $\left(M(\mathrm{~B})_{\beta}^{*}, \star\right)$ are unital Banach algebras, with respective identities $\epsilon$ and $\widetilde{\epsilon}$, and strict extension defines a unital isometric algebra isomorphism from the former to the latter.

Proof. Straightforward.

Definition. A convolution semigroup of functionals on B is a family of functionals $\left(\lambda_{t}\right)_{t \geq 0}$ in $\mathrm{B}^{*}$ satisfying

$$
\lambda_{0}=\epsilon \text { and } \lambda_{s+t}=\lambda_{s} \star \lambda_{t}, \quad s, t \geq 0 ;
$$

It is weakly continuous if

$$
\lim _{t \rightarrow 0^{+}} \lambda_{t}(a)=\epsilon(a), \quad a \in \mathrm{B}
$$

and norm-continuous if

$$
\lim _{t \rightarrow 0^{+}}\left\|\lambda_{t}-\epsilon\right\|=0 .
$$

Restricting to semigroups of states, Propositions 1.1 and 2.1 imply the following.

Proposition 2.2. Strict extension defines a one-to-one correspondence between weakly continuous convolution semigroups of states on $\mathrm{B}$ and weakly continuous convolution semigroups of strict states on $M(\mathrm{~B})$.

The norm-continuous case is summarised in the next result. In brief, normcontinuous convolution semigroups have bounded generators, from which the semigroup may be recovered by exponentiation. This also operates at the multiplier algebra level.

Proposition 2.3. Let $\left(\lambda_{t}\right)_{t \geq 0}$ be a norm-continuous convolution semigroup of functionals on $\mathrm{B}$. Then there is a unique functional $\gamma \in \mathrm{B}^{*}$ such that

$$
\lim _{t \rightarrow 0^{+}}\left\|\frac{1}{t}\left(\lambda_{t}-\epsilon\right)-\gamma\right\|=0 .
$$

Moreover,

$$
\lambda_{t}=\sum_{n=0}^{\infty} \frac{1}{n !}(t \gamma)^{\star n}, \quad \widetilde{\lambda}_{t}=\sum_{n=0}^{\infty} \frac{1}{n !}(t \widetilde{\gamma})^{\star n}, \quad t \geq 0
$$


and

$$
\lim _{t \rightarrow 0^{+}}\left\|\frac{1}{t}\left(\widetilde{\lambda}_{t}-\widetilde{\epsilon}\right)-\widetilde{\gamma}\right\|=0 .
$$

The functional $\gamma$ is called the generating functional of $\left(\lambda_{t}\right)_{t \geq 0}$.

Proof. In view of Proposition 2.1, this follows from elementary properties of normcontinuous semigroups in a unital Banach algebra.

The class of norm-continuous convolution semigroups of states is analogous to the class of classical convolution semigroups of compound Poisson type, with 'initial measure' $\gamma+\epsilon$. More justification for this terminology is given in [Fra] and [LS $\mathrm{L}_{1}$. The class of functionals $\gamma \in \mathrm{B}^{*}$ which generate a convolution semigroup of states on $\mathrm{B}$ is characterised as follows $\left(\left[\mathrm{LS}_{2}\right]\right): \gamma$ is $*$-preserving, conditionally positive in the sense that $\gamma(a) \geq 0$ for $a \in \mathrm{B}_{+} \cap \operatorname{Ker} \epsilon$ and satisfies $\widetilde{\gamma}(1)=0$. This is a form of Schönberg correspondence; see [Sch] for its algebraic (i.e. non-analytic) counterpart. For further classical motivations we refer to [Hey].

To each functional $\mu \in \mathrm{B}^{*}$ there are associated two completely bounded strict maps $\mathrm{B} \rightarrow M(\mathrm{~B})$ :

$$
L_{\mu}=\left(\mu \otimes \operatorname{id}_{\mathrm{B}}\right) \Delta \text { and } R_{\mu}=\left(\operatorname{id}_{\mathrm{B}} \otimes \mu\right) \Delta,
$$

and their strict extensions $\widetilde{L}_{\mu}, \widetilde{R}_{\mu}: M(\mathrm{~B}) \rightarrow M(\mathrm{~B})$. Note that the original functional may be recovered from either of these maps: $\epsilon \circ L_{\mu}=\mu=\epsilon \circ R_{\mu}$; from which it follows that $\left\|L_{\mu}\right\|_{\mathrm{cb}}=\left\|L_{\mu}\right\|=\|\mu\|=\left\|R_{\mu}\right\|=\left\|R_{\mu}\right\|_{\mathrm{cb}}$. If the map $L_{\mu}$ is positive then the functional $\mu$ is positive, so $L_{\mu}$ is actually completely positive; also $\widetilde{L}_{\mu}$ is unital if and only if $\widetilde{\mu}$ is. Thus $\widetilde{L}_{\mu}$ is completely positive and unital if and only if $\mu$ is a state. The same goes for the maps $R_{\mu}$ and $\widetilde{R}_{\mu}$. The strict extensions of the $L$-map $\mu \mapsto L_{\mu}$ and $R$-map $\mu \mapsto R_{\mu}$ define completely isometric unital algebra morphisms between the Banach algebras $\left(\mathrm{B}^{*}, \star\right)$ and $C B(M(\mathrm{~B}))$, sharing the same left inverse: $\widetilde{T} \mapsto \epsilon \circ T$, where $T:=\left.\widetilde{T}\right|_{\mathrm{B}}$.

For any convolution semigroup of functionals $\left(\lambda_{t}\right)_{t \geq 0}$ on B, $\left(\widetilde{P}_{t}:=\widetilde{R}_{\lambda_{t}}\right)_{t \geq 0}$ therefore defines a strict semigroup of completely bounded maps on $M(\mathrm{~B})$, which we call the associated semigroup on the multiplier algebra. It determines the original convolution semigroup via the identity:

$$
\widetilde{\lambda}_{t}=\widetilde{\epsilon} \circ \widetilde{P}_{t}, \quad t \geq 0 .
$$

Moreover, the semigroup $\left(\widetilde{P}_{t}\right)_{t \geq 0}$ is completely positive and unital if and only if $\left(\lambda_{t}\right)_{t \geq 0}$ is a convolution semigroup of states. We stress the point that, in the noncompact case (B nonunital), $P_{t}:=R_{\lambda_{t}}$ need not leave $\mathrm{B}$ invariant and so there may be no semigroup on $\mathrm{B}$ itself. In the next section we shall see that, under a natural condition on $B$, this obstruction is removed.

The semigroups on $M(\mathrm{~B})$ which are associated with convolution semigroups of functionals on B are characterised in several simple ways. This is the content of the next result. The convention on composing strict maps permits us to dispense with almost all tildes.

Theorem 2.4. Let $\left(\widetilde{P}_{t}\right)_{t \geq 0}$ be a strict semigroup on $M(\mathrm{~B})$ and set $P_{t}:=\left.\widetilde{P}_{t}\right|_{\mathrm{B}}$ : $\mathrm{B} \rightarrow M(\mathrm{~B})(t \geq 0)$. Then the following conditions are equivalent:

(i) $\left(\widetilde{P}_{t}\right)_{t \geq 0}$ is associated to a convolution semigroup of functionals on $\mathrm{B}$; 
(ii) $\left(P_{t}\right)_{t \geq 0}$ enjoys the commutativity property:

$$
L_{\mu} P_{t}=P_{t} L_{\mu}, \quad \mu \in \mathrm{B}^{*}, t \geq 0
$$

(iii) for each $t \geq 0, P_{t}$ is completely bounded, $\mathrm{id}_{\mathrm{B}} \otimes P_{t}$ is strict as a map from $\mathrm{B} \otimes \mathrm{B}$ to $M(\mathrm{~B} \otimes \mathrm{B})$, and the following strong invariance condition holds:

$$
\Delta P_{t}=\left(\operatorname{id}_{\mathrm{B}} \otimes P_{t}\right) \Delta
$$

(iv) $\left(P_{t}\right)_{t \geq 0}$ satisfies the weak invariance condition:

$$
P_{t}=\left(\operatorname{id}_{\mathrm{B}} \otimes\left(\epsilon \circ P_{t}\right)\right) \Delta, \quad t \geq 0 .
$$

Proof. The implication (iii) $\Rightarrow$ (ii) is immediate.

(ii) $\Rightarrow$ (iv): Assume that (ii) holds and let $t \geq 0$. Then, since $\epsilon \circ L_{\mu}=\mu$,

$$
\mu \circ\left(\operatorname{id}_{\mathrm{B}} \otimes\left(\epsilon \circ P_{t}\right)\right) \Delta=\left(\epsilon \circ P_{t}\right) L_{\mu}=\epsilon \circ\left(L_{\mu} P_{t}\right)=\mu \circ P_{t}, \quad \mu \in \mathrm{B}^{*},
$$

so (iv) holds.

(iv) $\Rightarrow(\mathrm{i})$ : Assume that (iv) holds and define $\lambda_{t}=\epsilon \circ P_{t} \in \mathrm{B}^{*}(t \geq 0)$. Then, for $s, t \geq 0$,

$$
\begin{aligned}
\left(\lambda_{s} \otimes \lambda_{t}\right) \Delta & =\left(\left(\epsilon \circ P_{s}\right) \otimes\left(\epsilon \circ P_{t}\right)\right) \Delta \\
& =\left(\epsilon \circ P_{s}\right)\left(\mathrm{id}_{\mathrm{B}} \otimes\left(\epsilon \circ P_{t}\right)\right) \Delta=\left(\epsilon \circ P_{s}\right) P_{t}=\epsilon \circ P_{s+t}=\lambda_{s+t},
\end{aligned}
$$

and

$$
R_{\lambda_{t}}=\left(\mathrm{id}_{\mathrm{B}} \otimes \lambda_{t}\right) \Delta=\left(\mathrm{id}_{\mathrm{B}} \otimes\left(\epsilon \circ P_{t}\right)\right) \Delta=P_{t} .
$$

Thus $\left(\lambda_{t}\right)_{t \geq 0}$ is a convolution semigroup of functionals on $\mathrm{B}$ and $\left(\widetilde{P}_{t}\right)_{t \geq 0}$ is its associated semigroup on $M(\mathrm{~B})$.

(i) $\Rightarrow\left(\right.$ iii): Assume that $\left(\widetilde{P}_{t}\right)_{t \geq 0}$ is the semigroup on $M(\mathrm{~B})$ associated with a convolution semigroup of functionals $\left(\lambda_{t}\right)_{t \geq 0}$ on $\mathrm{B}$, and let $t \geq 0$. Then $P_{t}$ is completely bounded, $\mathrm{id}_{\mathrm{B}} \otimes P_{t}$ equals the composition $\left(\mathrm{id}_{\mathrm{B}} \otimes \mathrm{id}_{\mathrm{B}} \otimes \lambda_{t}\right)\left(\mathrm{id}_{\mathrm{B}} \otimes \Delta\right)$ and so is strict, and (2.3) holds by coassociativity:

$$
\Delta P_{t}=\Delta\left(\mathrm{id}_{\mathrm{B}} \otimes \lambda_{t}\right) \Delta=\left(\mathrm{id}_{\mathrm{B}} \otimes \mathrm{id}_{\mathrm{B}} \otimes \lambda_{t}\right) \Delta^{(2)}=\left(\mathrm{id}_{\mathrm{B}} \otimes P_{t}\right) \Delta .
$$

Remarks. The above proof yields the following useful characterisations of the range of the $R$-map:

$$
\begin{aligned}
\operatorname{Ran} R & =\left\{T \in B(\mathrm{~B} ; M(\mathrm{~B})): T \text { is strict and } L_{\mu} T=T L_{\mu} \text { for all } \mu \in \mathrm{B}^{*}\right\} \\
& =\left\{T \in C B(\mathrm{~B} ; M(\mathrm{~B})):\left(\mathrm{id}_{\mathrm{B}} \otimes T\right) \text { is strict and } \Delta T=\left(\operatorname{id}_{\mathrm{B}} \otimes T\right) \Delta\right\} \\
& =\left\{T \in B(\mathrm{~B} ; M(\mathrm{~B})): T=\left(\operatorname{id}_{\mathrm{B}} \otimes(\epsilon \circ T)\right) \Delta\right\} .
\end{aligned}
$$

In the absence of a counit these equalities are replaced by

$$
\begin{aligned}
\operatorname{Ran} R & \subset\left\{T \in C B(\mathrm{~B} ; M(\mathrm{~B})):\left(\mathrm{id}_{\mathrm{B}} \otimes T\right) \text { is strict and } L_{\mu} T=T L_{\mu} \text { for all } \mu \in \mathrm{B}^{*}\right\} \\
& =\left\{T \in C B(\mathrm{~B} ; M(\mathrm{~B})):\left(\mathrm{id}_{\mathrm{B}} \otimes T\right) \text { is strict and } \Delta T=\left(\mathrm{id}_{\mathrm{B}} \otimes T\right) \Delta\right\} .
\end{aligned}
$$

The equality follows from the fact that the set $\left\{\left(\mu \otimes \mathrm{id}_{\mathrm{B}}\right)^{\sim}: \mu \in \mathrm{B}^{*}\right\}$ separates $M(\mathrm{~B} \otimes \mathrm{B})$, and the inclusion from the fact that $\left(\mu \otimes \mathrm{id}_{\mathrm{B}} \otimes \lambda\right) \Delta^{(2)}$ is a common expression for $L_{\mu} R_{\lambda}$ and $R_{\lambda} L_{\mu}\left(\lambda, \mu \in \mathrm{B}^{*}\right)$. 
So far we have not considered the question of continuity, in $t$, for convolution semigroups and their associated semigroups. It is easily seen that one is normcontinuous if and only the other is, in which case the generating functional and the generator of the associated semigroup are related by $Z_{\sim}=\widetilde{R}_{\gamma}$ and $\widetilde{\gamma}=\widetilde{\epsilon} \circ Z_{\sim}$. The corresponding statement at the level of weak/strong continuity need not be true. For example the translation semigroup on $C_{\mathrm{b}}(\mathbb{R})$ is the semigroup associated with the convolution semigroup of Dirac measures $\left(\delta_{t}\right)_{t>0}$ on $\mathbb{R}$. Whereas the latter is weakly continuous, the former is not. However the translation semigroup leaves $C_{0}(\mathbb{R})$ invariant and restricts to a strongly continuous semigroup there. This is a Feller property ([Jac]) which, in our framework, corresponds to the semigroup $\left(\widetilde{P}_{t}\right)_{t \geq 0}$ leaving the $C^{*}$-algebra B invariant and restricting to a $C_{0}$-semigroup on B. In the next section we shall see that this holds for all weakly continuous semigroups on a wide class of locally compact quantum semigroups which includes all locally compact (quantum) groups. We warn the reader that there is a variety of definitions of 'Feller' and 'strong Feller' - in both the classical and noncommutative literature (e.g. $[\mathrm{Sau}])$.

\section{Convolution Semigroups on $C^{*}$-Bialgebras Satisfying THE RESIDUAL VANISHING AT INFINITY CONDITION}

Throughout this section we assume that $\mathrm{B}$ is a $C^{*}$-bialgebra which enjoys the following 'residual vanishing at infinity' property:

$$
(\mathrm{B} \otimes 1) \Delta(\mathrm{B}) \subset \mathrm{B} \otimes \mathrm{B} \text { and }(1 \otimes \mathrm{B}) \Delta(\mathrm{B}) \subset \mathrm{B} \otimes \mathrm{B} .
$$

The $C^{*}$-bialgebras arising from locally compact quantum groups in the sense of Kustermans and Vaes $([\mathrm{KuV}])$ satisfy this property, as do (trivially) all unital $C^{*}$ bialgebras. In the classical case of a commutative quantum semigroup (see Section 5$), \mathrm{B}=C_{0}(H)$ for a locally compact semigroup $H, \mathrm{~B} \otimes \mathrm{B}$ and $M(\mathrm{~B} \otimes \mathrm{B})$ are identified with $C_{0}(H \times H)$ and $C_{\mathrm{b}}(H \times H)$ respectively, and the coproduct is given by $\Delta(F)\left(h_{1}, h_{2}\right)=F\left(h_{1} h_{2}\right)$, for $F \in \mathrm{B}$ and $h_{1}, h_{2} \in H$. To see how the condition applies then, let $F, F^{\prime} \in C_{0}(H)$. If $|F(h)|,\left|F^{\prime}(h)\right|<\epsilon$ for $h \in H \backslash K$ then $\left|F\left(h_{1}\right) F^{\prime}\left(h_{1} h_{2}\right)\right|<\epsilon^{2}$ for $\left(h_{1}, h_{2}\right) \in H \times H \backslash \phi^{-1}(K \times K)$ where $\phi$ is the homeomorphism $\left(h_{1}, h_{2}\right) \mapsto\left(h_{1}, h_{1} h_{2}\right)$. It follows that $(F \otimes 1) \Delta F^{\prime} \in C_{0}(H \times H)=\mathrm{B} \otimes \mathrm{B}$ and similarly for $(1 \otimes F) \Delta F^{\prime}$. Lance credits Iain Raeburn for the suggestive terminology (in $[\operatorname{Lan}]$ ).

The maps $R_{\mu}$ and $L_{\mu}$ defined in (2.1) now act on $\mathrm{B}$, as we show next.

Proposition 3.1. Let $\mu \in \mathrm{B}^{*}$. Then

$$
R_{\mu}(\mathrm{B}) \subset \mathrm{B} \text { and } L_{\mu}(\mathrm{B}) \subset \mathrm{B} .
$$

Proof. This follows from the fact, commonly used in topological quantum group theory, that $\mu \in \mathrm{B}^{*}$ may be factorised as $\lambda c$, for some $\lambda \in \mathrm{B}^{*}$ and $c \in \mathrm{B}$ ([Tay]);

$$
\begin{aligned}
L_{\mu}(a) & =\left(\mu \otimes \operatorname{id}_{\mathrm{B}}\right)^{\sim}(\Delta(a)) \\
& =\left(\lambda \otimes \operatorname{id}_{\mathrm{B}}\right)^{\sim}((c \otimes 1) \Delta(a)) \in\left(\lambda \otimes \operatorname{id}_{\mathrm{B}}\right)(\mathrm{B} \otimes \mathrm{B}) \subset \mathrm{B}, \quad a \in \mathrm{B},
\end{aligned}
$$

and similarly for $R_{\mu}$.

The significance of this is that each convolution semigroup of functionals $\left(\lambda_{t}\right)_{t \geq 0}$ on B now has an associated semigroup on the $C^{*}$-algebra B itself: $\left(P_{t}:=R_{\lambda_{t}}\right)_{t \geq 0}$. 
Reconstruction of the convolution semigroup from its associated semigroup now reads:

$$
\lambda_{t}=\epsilon \circ P_{t}, \quad t \geq 0,
$$

and Theorem 2.4 now has the following version.

Proposition 3.2. Let $\left(P_{t}\right)_{t \geq 0}$ be a strict semigroup on $\mathrm{B}$. Then the following conditions are equivalent:

(i) $\left(P_{t}\right)_{t \geq 0}$ is associated to a convolution semigroup of functionals on B;

(ii) $\left(P_{t}\right)_{t \geq 0}$ enjoys the commutativity property

$$
L_{\mu} P_{t}=P_{t} L_{\mu}, \quad \mu \in \mathrm{B}^{*}, t \geq 0 ;
$$

(iii) for each $t \geq 0, P_{t}$ is completely bounded, $\mathrm{id}_{\mathrm{B}} \otimes P_{t}$ is strict and the following strong invariance condition holds:

$$
\Delta P_{t}=\left(\mathrm{id}_{\mathrm{B}} \otimes P_{t}\right) \Delta .
$$

(iv) $\left(P_{t}\right)_{t \geq 0}$ satisfies the weak invariance condition:

$$
P_{t}=\left(\operatorname{id}_{\mathrm{B}} \otimes\left(\epsilon \circ P_{t}\right)\right) \Delta, \quad t \geq 0 ;
$$

Moreover we now have a satisfactory correspondence between continuity properties of the respective semigroups, showing that the semigroup on $M(\mathrm{~B})$ associated with any weakly continuous convolution semigroup of functionals on B is necessarily Feller.

Theorem 3.3. Let $\left(P_{t}\right)_{t \geq 0}$ be the semigroup on $\mathrm{B}$ associated with a convolution semigroup of functionals $\left(\lambda_{t}\right)_{t \geq 0}$ on $\mathrm{B}$. Then the following are equivalent:

(i) $\left(\lambda_{t}\right)_{t \geq 0}$ is weakly continuous;

(ii) $\left(P_{t}\right)_{t \geq 0}$ is a $C_{0}$-semigroup.

Proof. This follows from the identities

$$
\left(\lambda_{t}-\epsilon\right)=\epsilon \circ\left(P_{t}-\mathrm{id}_{\mathrm{B}}\right) \text { and } \mu \circ\left(P_{t}-\mathrm{id}_{\mathrm{B}}\right)=\left(\lambda_{t}-\epsilon\right) \circ L_{\mu},
$$

for $t \geq 0$ and $\mu \in \mathrm{B}^{*}$.

Under the weak continuity assumption, Proposition 3.2 can be formulated at the level of generators.

Proposition 3.4. Let $\left(P_{t}\right)_{t \geq 0}$ be a $C_{0}$-semigroup on $\mathrm{B}$ with generator $Z$. Then the following are equivalent:

(i) $\left(P_{t}\right)_{t \geq 0}$ is the semigroup associated with a weakly continuous convolution semigroup of functionals;

(ii) $Z$ enjoys the commutativity property:

$$
L_{\mu} Z \subset Z L_{\mu}, \quad \mu \in \mathrm{B}^{*},
$$

that is, $L_{\mu}(\operatorname{Dom} Z) \subset \operatorname{Dom} Z$ and $L_{\mu} Z(a)=Z L_{\mu}(a)$ for all $a \in \operatorname{Dom} Z$.

Proof. This follows from Theorem 3.3, the equivalence (i) $\Leftrightarrow$ (ii) of Proposition 3.2, and Theorem 1.15 of [Dav]. 
Definition. For a weakly continuous convolution semigroup of functionals $\left(\lambda_{t}\right)_{t \geq 0}$ on $\mathrm{B}$, the functional $\gamma: \operatorname{Dom} \gamma \subset \mathrm{B} \rightarrow \mathbb{C}$ defined by

$$
\begin{aligned}
& \operatorname{Dom} \gamma:=\left\{a \in \mathrm{B}: \lim _{t \rightarrow 0^{+}} \frac{\lambda_{t}(a)-\epsilon(a)}{t} \text { exists }\right\} ; \\
& \gamma(d):=\lim _{t \rightarrow 0^{+}} \frac{\lambda_{t}(d)-\epsilon(d)}{t}, \quad d \in \operatorname{Dom} \gamma,
\end{aligned}
$$

is called the generating functional of $\left(\lambda_{t}\right)_{t \geq 0}$.

Remarks. If $\left(\lambda_{t}\right)_{t \geq 0}$ is norm-continuous then the functional $\gamma$ defined above is equal to that of Proposition 2.3, so our terminology is consistent. If each $\lambda_{t}$ is ${ }^{*}$-preserving then $\operatorname{Dom} \gamma$ is selfadjoint and $\gamma$ is ${ }^{*}$-preserving. If each $\lambda_{t}$ is a state, then $\gamma$ is conditionally positive, in the sense that $\gamma(a) \geq 0$ for all $a \in \mathrm{B}_{+} \cap \operatorname{Dom} \gamma \cap \operatorname{Ker} \epsilon$ (again, consistently with the earlier definition). The identity (3.1) implies the inclusion $\epsilon \circ Z \subset \gamma$, where $Z$ is the generator of the associated $C_{0}$-semigroup on $\mathrm{B}$, so Dom $\gamma$ is norm-dense in B.

Note that $\gamma$ can be defined for a weakly continous convolution semigroup on any $C^{*}$-bialgebra, however, without the residual vanishing at infinity property, there is no guarantee that $\gamma$ be densely defined.

We now recall a useful construction from semigroup theory. Let $\left(P_{t}\right)_{t \geq 0}$ be a $C_{0}$-semigroup on a Banach space $X$ with generator $Z$. Set

$$
\operatorname{Dom} \widehat{Z}:=\left\{x \in X: \forall_{\mu \in X^{*}} \lim _{t \rightarrow 0^{+}} \frac{\mu\left(P_{t}(x)\right)-\mu(x)}{t} \text { exists }\right\} .
$$

Clearly Dom $\widehat{Z} \supset \operatorname{Dom} Z$. By the Uniform Boundedness Principle, it follows that, for all $x \in \operatorname{Dom} \widehat{Z}$, there is a unique element $l \in X^{* *}$ satisfying

$$
\lim _{t \rightarrow 0^{+}} \frac{\mu\left(P_{t}(x)\right)-\mu(x)}{t}=l(\mu), \quad \mu \in X^{*} .
$$

Setting $\widehat{Z}(x):=l$, we have an operator $\widehat{Z}: X \rightarrow X^{* *}$ with dense domain $\operatorname{Dom} \widehat{Z}$ (justifying the notation (3.2)). Under the canonical identification of $X$ as a subspace of $X^{* *}$, clearly $\widehat{Z} \supset Z$. The precise situation is summarised next. This is a rewording of Theorem 1.24 of [Dav], where the reader is warned of a missing hypothesis (not needed here), namely that -in the notation used there- $\mathcal{L}$ must also be assumed to be invariant under each $T_{t}^{*}$.

Lemma 3.5. Let $\left(P_{t}\right)_{t \geq 0}$ be a $C_{0}$-semigroup on a Banach space $X$ with generator $Z$, and associated operator $\widehat{Z}: \operatorname{Dom} \widehat{Z} \subset X \rightarrow X^{* *}$. Then

$$
\operatorname{Dom} Z=\{x \in \operatorname{Dom} \widehat{Z}: \widehat{Z}(x) \in X\} \text { and } Z=\left.\widehat{Z}\right|_{\operatorname{Dom} Z} .
$$

In particular, the operator $\widehat{Z}$ uniquely determines the semigroup $\left(P_{t}\right)_{t \geq 0}$.

The significance of the operator $\widehat{Z}$ for present considerations is indicated by the following result.

Proposition 3.6. Let $\left(P_{t}\right)_{t \geq 0}$ be the semigroup on B associated with a weakly continuous convolution semigroup of functionals $\left(\lambda_{t}\right)_{t \geq 0}$ on $\mathrm{B}$. Then its associated operator $\widehat{Z}: \operatorname{Dom} \widehat{Z} \subset \mathrm{B} \rightarrow \mathrm{B}^{* *}$ is given by

$$
\begin{aligned}
& \operatorname{Dom} \widehat{Z}=\left\{a \in \mathrm{B}: \forall_{\mu \in \mathrm{B}^{*}} L_{\mu}(a) \in \operatorname{Dom} \gamma\right\} ; \text { and } \\
& \widehat{Z}(a)(\mu)=\gamma\left(L_{\mu}(a)\right), \quad a \in \operatorname{Dom} \widehat{Z}, \mu \in \mathrm{B}^{*},
\end{aligned}
$$


where $\gamma$ is the generating functional of $\left(\lambda_{t}\right)_{t \geq 0}$.

Proof. If $\mu \in \mathrm{B}^{*}, a \in \mathrm{B}$ and $t \geq 0$ then $\mu\left(P_{t}(a)\right)=\lambda_{t}\left(L_{\mu}(a)\right)$; in particular $\mu(a)=$ $\epsilon\left(L_{\mu}(a)\right)$. The identities (3.4) and (3.5) now follow directly from the definitions of $\gamma$ and $\widehat{Z}$.

The next result, the first part of which fully justifies the term 'generating functional', now follows easily from Proposition 3.6, Lemma 3.5 and the identity (3.1).

Theorem 3.7. Let $\left(\lambda_{t}\right)_{t \geq 0}$ be a weakly continuous convolution semigroup of functionals on $\mathrm{B}$ with generating functional $\gamma$. Then

(a) the functional $\gamma$ determines the semigroup $\left(\lambda_{t}\right)_{t \geq 0}$ uniquely;

(b) $\gamma$ is bounded if and only if $\left(\lambda_{t}\right)_{t \geq 0}$ is norm-continuous, in which case $\gamma$ is everywhere defined.

\section{Convolution semigroups on $C^{*}$-Bialgebras of Discrete type}

For this section we assume that $\mathrm{B}$ is of discrete type, that is, as a $C^{*}$-algebra, $\mathrm{B}$ is a $c_{0}$-direct sum of matrix algebras. The existence of a counit forces $B$ to have the form

$$
\mathrm{B}=\mathbb{C} \Omega \oplus \mathrm{B}_{0} \text { where } \mathrm{B}_{0}=\bigoplus_{\lambda \in \Lambda} M_{n(\lambda)},
$$

for an index set $\Lambda$ and family $(n(\lambda))_{\lambda \in \Lambda}$ in $\mathbb{N}$; the counit being given by the formula

$$
\epsilon\left(\alpha \Omega \oplus \bigoplus_{\lambda \in \Lambda} a_{\lambda}\right)=\alpha .
$$

We identify $\mathrm{B}_{0}$ with $0 \oplus \mathrm{B}_{0} \subset \mathrm{B}$ and view $\mathrm{B}$ as represented on the Hilbert space $\mathbb{C} \oplus \bigoplus_{\lambda \in \Lambda} \mathbb{C}^{n(\lambda)}$. Then $M(\mathrm{~B})$ equals the $l^{\infty}$-direct sum

$$
\mathrm{M}:=\mathbb{C} \Omega \oplus \prod_{\lambda \in \Lambda} M_{n(\lambda)},
$$

which is a von Neumann algebra, coinciding with the universal enveloping von Neumann algebra of $B$.

The examples we have in mind are the algebras of functions vanishing at infinity on a discrete semigroup, and the duals of compact (quantum) groups. In the former case $n(\lambda)=1$ for each $\lambda \in \Lambda$; in the latter case $\Lambda$ is the set of equivalence classes of irreducible representations of the underlying compact group; more on these in the next section.

Lemma 4.1. Let $\left(\lambda_{t}\right)_{\geq 0}$ be a weakly continuous convolution semigroup of states on $\mathrm{B}$. Then its associated semigroup $\left(\widetilde{P}_{t}\right)_{t \geq 0}$ on $M(\mathrm{~B})$ is strongly continuous.

Proof. Since $M(\mathrm{~B})$ is the von Neumann algebra $\mathrm{M}$, and by the same token $M(\mathrm{~B} \otimes \mathrm{B})$ is the von Neumann algebra $M \bar{\otimes} \mathrm{M}$, Proposition 1.2 gives maps

$$
L_{\nu}:=\left(\nu \otimes_{\mathrm{M}} \mathrm{id}_{\mathrm{M}}\right) \widetilde{\Delta}: M(\mathrm{~B}) \rightarrow M(\mathrm{~B}), \quad \nu \in M(\mathrm{~B})^{*}=\mathrm{M}^{*},
$$

satisfying

$$
\tilde{\lambda} \circ L_{\nu}=\nu \circ\left(\operatorname{id}_{\mathrm{M}} \bar{\otimes} \tilde{\lambda}\right) \widetilde{\Delta}=\nu \circ \widetilde{R}_{\lambda}, \quad \lambda \in \mathrm{B}^{*}=\mathrm{M}_{*}, \nu \in M(\mathrm{~B})^{*}=\mathrm{M}^{*} .
$$

In particular,

$$
\nu \circ\left(\widetilde{P}_{t}-\operatorname{id}_{M(\mathrm{~B})}\right)=\left(\widetilde{\lambda}_{t}-\widetilde{\epsilon}\right) \circ L_{\nu}, \quad t \geq 0, \nu \in M(\mathrm{~B})^{*} .
$$


Therefore, by Proposition 2.2, $\left(\widetilde{P}_{t}\right)_{t \geq 0}$ is weakly continuous, and so also strongly continuous.

Remark. Note how the existence of slice maps for not-necessarily-strict functionals is crucially used in the above proof (via Proposition 1.2).

Theorem 4.2. Let $\left(\lambda_{t}\right)_{t \geq 0}$ be a weakly continuous convolution semigroup of states on $\mathrm{B}$. Then $\left(\lambda_{t}\right)_{t \geq 0}$ is norm-continuous.

Proof. Define a functional $\gamma_{\sim}: \operatorname{Dom} \gamma_{\sim} \subset M(B) \rightarrow \mathbb{C}$ by

$$
\begin{aligned}
& \operatorname{Dom} \gamma_{\sim}:=\left\{m \in M(\mathrm{~B}): \lim _{t \rightarrow 0^{+}} \frac{\widetilde{\lambda}_{t}(m)-\widetilde{\epsilon}(m)}{t} \text { exists }\right\} ; \\
& \gamma_{\sim}(d)=\lim _{t \rightarrow 0^{+}} \frac{\widetilde{\lambda}_{t}(d)-\widetilde{\epsilon}(d)}{t}, \quad d \in \operatorname{Dom} \gamma_{\sim} .
\end{aligned}
$$

Clearly Dom $\gamma_{\sim}$ is a selfadjoint subspace of $M(\mathrm{~B})$ containing 1 and $\gamma_{\sim}(1)=0$. Moreover, the identity (2.2) and Lemma 4.1 imply that Dom $\gamma_{\sim}$ is norm-dense in $M(\mathrm{~B})$ (cf. the remarks in Section 3).

Let $p$ be the orthogonal projection $1-\Omega \in M(\mathrm{~B})$. Note that $p$ is the identity of the ideal $\operatorname{Ker} \widetilde{\epsilon}=M\left(\mathrm{~B}_{0}\right)$ of $M(\mathrm{~B})$. By density and selfadjointness of Dom $\gamma_{\sim}$ there exists $z \in \operatorname{Dom} \gamma_{\sim}$ such that $z=z^{*}$ and $\|p-z\| \leq \frac{1}{4}$. Note that $|\widetilde{\epsilon}(z)|=$ $|\widetilde{\epsilon}(z-p)| \leq \frac{1}{4}$. Put $y=z-\epsilon(z) 1$. Then $y \in \operatorname{Dom} \gamma_{\sim}$ and $\widetilde{\epsilon}(y)=0$, so in particular $\sup _{t>0} \frac{\tilde{\lambda}_{t}(y)}{t}<\infty$. Moreover, since $y \in M\left(\mathrm{~B}_{0}\right)$ and $z \geq p-\frac{1}{4} 1$,

$$
y=p y p=(p z p-\widetilde{\epsilon}(z) p) \geq p\left(p-\frac{1}{4} 1\right) p-\frac{1}{4} p=\frac{1}{2} p .
$$

Therefore

$$
C:=\sup _{t>0} \frac{\tilde{\lambda}_{t}(p)}{t} \leq 2 \sup _{t>0} \frac{\tilde{\lambda}_{t}(y)}{t}<\infty .
$$

Now, for each $t>0$, define a positive functional $\mu_{t}$ in $M(\mathrm{~B})_{\beta}^{*}$ by the formula

$$
\mu_{t}(m)=\frac{1}{t} \widetilde{\lambda}_{t}(p m p), \quad m \in M(\mathrm{~B}) .
$$

All these functionals have norm smaller than $C$ :

$$
\left\|\mu_{t}\right\|=\mu_{t}(1)=\frac{1}{t} \widetilde{\lambda}_{t}(p) \leq C .
$$

Now let $\nu$ be an arbitrary functional in $M(\mathrm{~B})^{*}$. Then, since $p$ is the identity element of $M\left(\mathrm{~B}_{0}\right)$, it follows from (4.2) that, for all $m \in M(\mathrm{~B})$,

$$
\begin{aligned}
\nu\left(\widetilde{P}_{t}(m)-m\right) & =\widetilde{\lambda}_{t}\left(L_{\nu}(m)-\widetilde{\epsilon}\left(L_{\nu}(m)\right) 1\right) \\
& =\widetilde{\lambda}_{t}\left(p\left(L_{\nu}(m)-\widetilde{\epsilon}\left(L_{\nu}(m)\right) 1\right) p\right)=t \mu_{t}\left(L_{\nu}(m)-\widetilde{\epsilon}\left(L_{\nu}(m)\right) 1\right) .
\end{aligned}
$$

Thus, letting $Z_{\sim}$ be the generator of the $C_{0}$-semigroup $\left(\widetilde{P}_{t}\right)_{t \geq 0}$,

$$
\begin{aligned}
\left|\nu\left(Z_{\sim}(m)\right)\right| & =\lim _{t \rightarrow 0^{+}} \frac{1}{t}\left|\nu\left(\widetilde{P}_{t}(m)-m\right)\right| \\
& \leq 2 C\left\|L_{\nu}(m)\right\| \leq 2 C\|\nu\|\|m\|, \quad m \in \operatorname{Dom} Z_{\sim} .
\end{aligned}
$$


It follows that $Z_{\sim}$ is bounded, so $\left(\widetilde{P}_{t}\right)_{t \geq 0}$ is norm-continuous, and therefore $\left(\lambda_{t}\right)_{t \geq 0}$ is too.

Remark. Since the multiplier algebra $M(\mathrm{~B})$ is a von Neumann algebra, Theorem 4.2 can alternatively be deduced from Lemma 4.1 using the fact that any strongly continuous completely positive semigroup on a von Neumann algebra is automatically norm-continuous ([Ell]).

We note here that the results of Sections 2, 3 and 4 remain valid for a $C^{*}$ hyperbialgebra $\mathrm{B}$, where the coproduct is only assumed to be completely positive, strict and preunital. Examples of $C^{*}$-hyperbialgebras are provided by compact quantum hypergroups $([\mathrm{ChV}])$. The classical theory is described in $[\mathrm{BlH}]$.

\section{Commutative and cocommutative Cases}

Let $\mathrm{B}$ be a commutative $C^{*}$-bialgebra of discrete type. Then it follows from Gelfand theory that $\mathrm{B}$ is of the form $C_{0}(\Gamma)$, where $\Gamma$ is a discrete semigroup, and the coproduct and counit are given by

$$
\Delta(F)\left(\gamma, \gamma^{\prime}\right)=f\left(\gamma \gamma^{\prime}\right) \text { and } \epsilon(F)=F(e), \quad F \in C_{0}(\Gamma), \gamma, \gamma^{\prime} \in \Gamma,
$$

under the natural identifications $M(\mathrm{~B} \otimes \mathrm{B})=M\left(C_{0}(\Gamma \times \Gamma)\right)=C_{\mathrm{b}}(\Gamma \times \Gamma)$, moreover the residual vanishing at infinity property holds, as mentioned earlier. In this case convolution semigroups of states on B correspond to convolution semigroups of probability measures on $\Gamma$ via the Riesz Representation Theorem, and Theorem 4.2 specialises as follows.

Proposition 5.1. Every convolution semigroup of probability measures on a discrete semigroup has bounded infinitesimal generator.

This extends the central conclusion of Theorem 4.1.5 of [Hey], which is formulated for discrete groups.

Recall that cocommutativity for a $C^{*}$-bialgebra $\mathrm{B}$ means $\Sigma \Delta=\Delta$ where $\Sigma$ denotes the tensor flip on $\mathrm{B} \otimes \mathrm{B}$ (which is strict as a map $\mathrm{B} \otimes \mathrm{B} \rightarrow M(\mathrm{~B} \otimes \mathrm{B})$ ); it is equivalent to commutativity of the convolution product on $B^{*}$. For a locally compact group $G$, the reduced and universal group $C^{*}$-algebras are isomorphic, via the left regular representation, if and only if $G$ is amenable ([Ped], Theorem 7.3.9), in particular this holds if $G$ is compact. Therefore, by the Peter-Weyl theory of unitary representations of compact groups, the group $C^{*}$-algebra of a compact group is isomorphic to a $c_{0}$-direct sum of matrix algebras.

We are not aware of any characterisation of cocommutative $C^{*}$-bialgebras of discrete type. However every cocommutative discrete quantum group B is the group $C^{*}$-algebra of a compact group $G$ where, viewing $C^{*}(G)$ as the $C^{*}$-subalgebra of $B\left(L^{2}(G\right.$, Haar $\left.)\right)$ generated by the set of convolution operators $\lambda(F): \xi \mapsto F \star \xi$ $(F \in C(G))$, the coproduct and counit are determined by

$$
\widetilde{\Delta}\left(\lambda_{g}\right)=\lambda_{g} \otimes \lambda_{g} \text { and } \widetilde{\epsilon}\left(\lambda_{g}\right)=1, \quad g \in G,
$$

$\lambda_{g} \in M(\mathrm{~B})$ being the translation operator $\left(\lambda_{g} \xi\right)\left(g^{\prime}\right)=\xi\left(g^{-1} g^{\prime}\right)$. This follows from duality theory for locally compact quantum groups ([BaS], $\left.\left[\mathrm{Ku}_{2}\right]\right)$. Specifically, discrete quantum groups are naturally isomorphic to their quantum group biduals; the dual of a cocommutative discrete quantum group is a commutative compact quantum group; and the dual of a commutative quantum group $C(G)$ is the group $C^{*}$-algebra of $G$ with the quantum group structure defined above. 
It is easily verified that the unital *-algebra spanned by $\left\{\lambda_{g}: g \in G\right\}$ is strictly dense in the multiplier algebra $M(\mathrm{~B})$, and that the map $g \mapsto \lambda_{g}$ is strictly continuous $G \rightarrow M(\mathrm{~B})$. The following consequence of Proposition 7.1.9 of [Ped], is noted here for convenience.

Proposition 5.2. Let B be the group $C^{*}$-algebra of a compact group $G$. Then

$$
\widetilde{\omega}\left(\lambda_{g}\right)=\phi(g), \quad g \in G,
$$

describes a one-to-one correspondence between the positive linear functionals $\omega$ on $\mathrm{B}$ and the continuous positive-definite functions $\phi$ on $G$.

Given a positive-definite function $\phi$ on a group $G$, the function $\psi: G \rightarrow \mathbb{C}$, $g \mapsto \phi(g)-\phi(e)$ plainly enjoys the following three properties: it is conditionally positive definite, that is, for all $n \in \mathbb{N}, g_{1}, \ldots, g_{n} \in G$ and $z_{1}, \ldots, z_{n} \in \mathbb{C}$ satisfying $\sum_{i=1}^{n} z_{i}=0$

$$
\sum_{i, j=1}^{n} \overline{z_{i}} z_{j} \psi\left(g_{i}^{-1} g_{j}\right) \geq 0
$$

it is Hermitian, i.e.

$$
\psi=\psi^{*} \text { where } \psi^{*}(g):=\overline{\psi\left(g^{-1}\right)}, \quad g \in G,
$$

and it vanishes at the identity element $e$ :

$$
\psi(e)=0 .
$$

The content of the theorem below is that the converse holds if $G$ is compact and $\psi$ is continuous. We deduce this from our results.

Theorem 5.3 ([Gui], Theorem 4.1). Let $G$ be a compact group and let $\psi \in C(G)$ be Hermitian, conditionally positive-definite and vanish at the identity element $e$. Then there is a positive-definite function $\phi$ on $G$ such that

$$
\psi(g)=\phi(g)-\phi(e), \quad g \in G,
$$

in other words there is a constant $C \in \mathbb{R}_{+}$such that $(\psi+C)$ is positive-definite.

Proof. Let B be the discrete quantum group $C^{*}(G)$, as above, and let $\mathcal{B}$ be the *-algebraic span of $\left\{\lambda_{g}: g \in G\right\}$ in $M(\mathrm{~B})$. By the Schönberg correspondence, the continuous function $e^{t \psi}$ is positive-definite for each $t \geq 0$ ([PaS $]$, Lemma 1.7). Let $\left(\mu_{t}\right)_{t \geq 0}$ be the corresponding family of states on B, defined via (5.1). Then, for all $g \in G$ and $s, t \in \mathbb{R}_{+}$,

$$
\left(\widetilde{\mu}_{s} \star \widetilde{\mu}_{t}\right)\left(\lambda_{g}\right)=\widetilde{\mu}_{s}\left(\lambda_{g}\right) \widetilde{\mu}_{t}\left(\lambda_{g}\right)=e^{s \psi(g)} e^{t \psi(g)}=e^{(s+t) \psi(g)}=\widetilde{\mu}_{s+t}\left(\lambda_{g}\right)
$$

and

$$
\widetilde{\mu}_{0}\left(\lambda_{g}\right)=1=\widetilde{\epsilon}\left(\lambda_{g}\right) .
$$

By the strict density of $\mathcal{B}$ in $M(\mathrm{~B})$, it follows that $\left(\mu_{t}\right)_{t \geq 0}$ is a convolution semigroup of states on $B$. In view of the identity

$$
\mu_{t}(\lambda(F))=\int_{G} F(g) e^{t \psi(g)} d g, \quad t \in \mathbb{R}_{+}, F \in C(G),
$$


it follows from the compactness of $G$ and Lebesgue's Dominated Convergence Theorem, that $\left(\mu_{t}\right)_{t \geq 0}$ is weakly continuous. Therefore, by Theorem 4.2, it is normcontinuous and so, by Proposition 2.3, the strict extension of its generating functional $\gamma$ satisfies

$$
\widetilde{\gamma}\left(\lambda_{g}\right)=\lim _{t \rightarrow 0^{+}} t^{-1}\left(\widetilde{\mu}_{t}\left(\lambda_{g}\right)-\widetilde{\epsilon}\left(\lambda_{g}\right)\right)=\lim _{t \rightarrow 0^{+}} t^{-1}\left(e^{t \psi(g)}-1\right)=\psi(g), \quad g \in G .
$$

By Theorem 6.3 of $\left[\mathrm{LS}_{2}\right]$, there is a nondegenerate representation $(\pi, \mathrm{h})$ of $\mathrm{B}$ and vector $\eta \in \mathrm{h}$ such that $\gamma=\omega_{\eta} \circ(\pi-\iota \circ \epsilon)$ where $\iota$ is the ampliation $\mathbb{C} \rightarrow B(\mathrm{~h})$ and $\omega_{\eta}$ denotes the vector functional $T \mapsto\langle\eta, T \eta\rangle$ on $B(\mathrm{~h})$. Letting $\phi: G \rightarrow \mathbb{C}$ be the positive-definite function $g \mapsto\left(\omega_{\eta} \circ \widetilde{\pi}\right)\left(\lambda_{g}\right)$, we have $\phi(g)-\phi(e)=\widetilde{\gamma}\left(\lambda_{g}\right)=\psi(g)$ for all $g \in G$, and so the proof is complete.

Remarks. Guichardet's approach is to reduce the proposition to the vanishing of the first cohomology group of unitary representations of compact groups and then to appeal to Theorem 15.1 of [PaS], which delivers just that.

Conversely, for the special case of multiplier $C^{*}$-bialgebras of the form $C^{*}(G)$ where $G$ is a compact group, Theorem 5.3 can be used to give an alternative proof of Theorem 6.3 of $\left[\mathrm{LS}_{2}\right]$

Acknowledgement. We are grateful to Uwe Franz for useful remarks on an earlier draft, in particular for bringing Guichardet's Theorem to our attention.

\section{REFERENCES}

[BaS] S. Baaj and G. Skandalis, Unitaires multiplicatifs commutatifs, C.R. Math. Acad. Sci. Paris 336 (2003), no. 4, 299-304.

[Ber] J. Bertoin, "Lévy Processes," Cambridge Tracts in Mathematics 121, CUP, 1996.

$[\mathrm{BlH}]$ W.R. Bloom and H. Heyer, "The Harmonic Analysis of Probability Measures on Hypergroups," de Gruyter, Berlin, 1995.

[ChV] Yu. Chapovsky and L. Vainerman, Compact quantum hypergroups, J. Operator Theory 41 (1999) no. 2, 261-289.

[Dav] E.B. Davies, "One-Parameter Semigroups," London Mathematical Society Monographs 15, Academic Press, London, 1980.

[Ell] G. Elliott, On the convergence of a sequence of completely positive maps to the identity, J. Austral. Math. Soc. Ser. A 68 (2000) no. 3, 340-348.

[Fra] U. Franz, Lévy processes on quantum groups and dual groups, in "Quantum Independent Increment Processes, Vol. II: Structure of Quantum Lévy Processes, Classical Probability and Physics," LNM 1866, Springer, Heidelberg, 2005.

[Gui] A. Guichardet, "Symmetric Hilbert Spaces and Related Topics," Lecture Notes in Mathematics 267, Springer, Heidelberg, 1970.

[Hey] H. Heyer, "Probability Measures on Locally Compact Groups," Springer-Verlag, Berlin, 1977.

[Jac] N. Jacob, "Pseudo-differential Operators and Markov Processes, Vol. I: Fourier Analysis and Semigroups," Imperial College Press, London, 2001.

[KaR] R.V. Kadison and J.R. Ringrose, "Fundamentals of the theory of operator algebras, Vol. II: Advanced Theory," Graduate Studies in Mathematics 16, AMS, 1997.

$\left[\mathrm{Ku}_{1}\right]$ J. Kustermans, One-parameter representations on $C^{*}$-algebras, Preprint, Odense Universitet, arXiv: \#funct-an/9707010.

$\left[\mathrm{Ku}_{2}\right]$ - - Locally compact quantum groups, in, "Quantum Independent Increment Processes, Vol. I: From Classical Probability to Quantum Stochastics," eds. U. Franz ES M. Schürmann, Lecture Notes in Mathematics 1865, Springer, Heidelberg, 2005.

$[\mathrm{KuV}]$ J. Kustermans and S. Vaes, Locally compact quantum groups, Ann. Sci. École Norm. Sup. (4) 33 (2000) no. 6, 837-934.

[Lan] E.C. Lance, "Hilbert $C^{*}$-modules," London Mathematical Society Lecture Notes Series 210, Cambridge University Press, Cambridge, 1995. 
[LS $]$ J.M. Lindsay and A.G. Skalski, Quantum stochastic convolution cocycles II, Comm. Math. Phys. 280 (2008) no. 3, 575-610;

[LS 2$]$ - - Quantum stochastic convolution cocycles III, Preprint, 2009.

[LiW] J.M. Lindsay and S.J.Wills, Existence of Feller cocycles on a $C^{*}$-algebra, Bull. London Math. Soc. 33 (2001) no. 5, 613-621.

[Neu] M. Neufang, Amplification of completely bounded operators and Tomiyama's slice maps, J. Funct. Anal. 207 (2004), 300-329.

[PaS] K.R. Parthasarathy and K. Schmidt, "Positive Definite Kernels, Continuous Tensor Products, and Central Limit Theorems of Probability Theory," Lecture Notes in Mathematics 272, Springer, Berlin, 1972.

[Ped] G.K. Pedersen, " $C^{*}$-algebras and their automorphism groups," London Mathematical Society Monographs 14, Academic Press, London New York, 1979.

[Sau] J.-L. Sauvageot, Strong Feller semigroups on $C^{*}$-algebras, J. Operator Theory 42 (1999) no. 1, 83-102.

[Sch] M.Schürmann, "White Noise on Bialgebras," Lecture Notes in Mathematics 1544, Springer, Heidelberg, 1993.

[Tay] D.C. Taylor, The strict topology for double centralizer algebras, Trans. Amer. Math. Soc. 150 (1970), 633-643.

[Tom] J. Tomiyama, Tensor products and approximation problems of $C^{*}$-algebras, Publ. RIMS, Kyoto Univ. 11 (1975), 163-183. 